\title{
The process of catalytic hydration of acetylene compounds using a homogeneous catalyst based on gold (I) complex with water-soluble organophosphorus ligands
}

\author{
Ivan Kokhan ${ }^{1}$, Sergiy Kudryavtsev $\mathrm{PhD}^{2}$ \\ 1. Faculty of Engineering, Volodymyr Dahl East Ukrainian National University, Ukraine, Sievierodonetsk, \\ Tsentralny Ave., 59a, E-mail: ivan.v.kokhan@gmail.com \\ 2. Faculty of Engineering, Volodymyr Dahl East Ukrainian National University, Ukraine, Sievierodonetsk, \\ Tsentralny Ave., 59a, E-mail: sergeykudryavtsevsnu@gmail.com
}

The work provides the description of one of the realizations of the process of catalytic hydration of acetylenic compounds using a homogeneous catalyst based on the gold (I) complex with water-soluble organophosphorus ligands. In particular, we are to present the process of catalytic hydration of acetylene. The reactor unit consists of a gas-liquid reactor and a column for the separation of the reaction product, acetaldehyde, is also is used. When developing the process, attention was paid to the peculiarities of using a homogeneous catalyst and preventing its entrainment from the reaction unit along with the reaction product.

Keywords - acetaldehyde, acetylene compounds, acetylene, homogeneous catalyst, gold complexes, catalytic hydration, organophosphorus ligands, gas-liquid reactor, product separation column.

\section{Introduction}

The last decades in the field of chemical engineering were marked by an active search for ways to use homogeneous metal complex catalysis in the processes of basic organic synthesis. There are several reasons for this, in particular, homogeneous metal complex catalysis can significantly increase the conversion level, process selectivity, while reducing energy consumption. The last of these factors is of particular interest, since the use of this type of catalysis allows the processes to be carried out at atmospheric or close to atmospheric pressure and at low temperatures. It is also worth noting that in many processes using homogeneous metal complex catalysis, with the correct selection of the ligand, it is possible to achieve the solubility of the catalyst in water, which in some cases makes it possible to abandon the use of organic solvents, or to reduce their use to a minimum. The above advantages are also largely characteristic of the process we are considering, the catalytic hydration of acetylene using catalyst based on the gold (I) complex. During a long time, industrial processes for the catalytic hydration of acetylenic compounds were based on the Kucherov reaction, in which mercury salts were used as a catalyst, which caused a number of problems associated with their toxicity. It should also be noted the need for a large amount of catalyst and the complexity of its regeneration, characteristic of the processes based on the Kucherov reaction. In connection with the above, the processes of hydration of acetylene compounds based on the Kucherov reaction are currently not used in the basic organic synthesis. These disadvantages of the above process were undertaken to search for new homogeneous metal complex catalysts, on the one hand, tested in preparative practice [1], on the other hand, those that can be used in the industrial process of catalytic hydration of acetylene. As a result of this research, the process described below has been developed.

\section{The process description}

The proposed process uses a homogeneous catalyst based on the gold (I) complex. The sodium triphenylphosphine trisulfonate (TPPTS) is used as a ligand. The counter ion is chlorine. 
The choice of the TPPTS as a ligand is due to the need to ensure the solubility of the catalyst in a water.

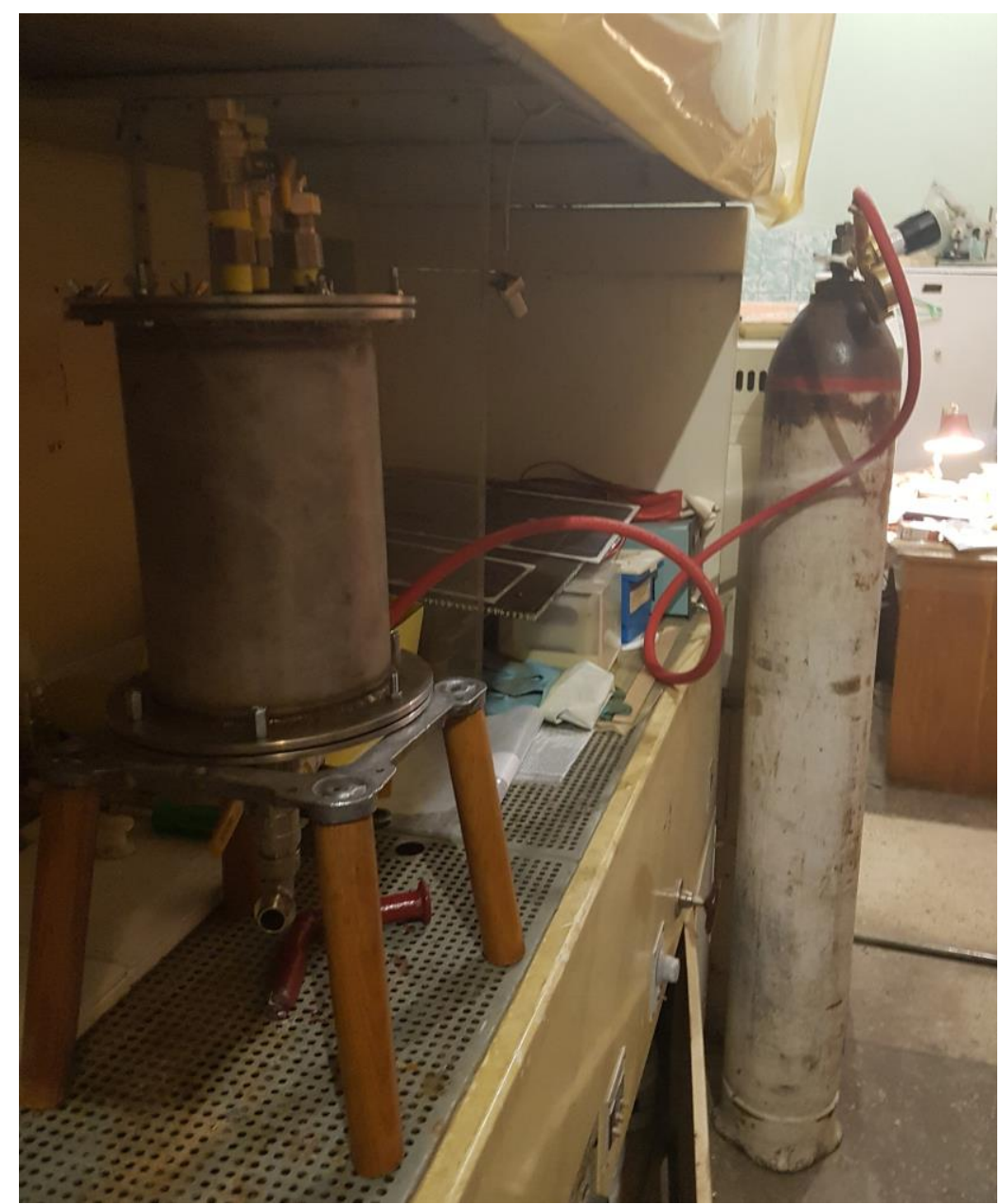

Fig.1. The pilot-laboratory installation for research the process of catalytic hydration of acetylene.

The reaction of catalytic hydration of acetylene is carried out in a gas-liquid reactor [2]. The reactor is filling of the water, which is both a solvent and a hydration reagent. The catalyst in an amount of about $0.2 \mathrm{~mol} \%$ is soluble in water. A small amount of sulfuric acid is used as a co-catalyst. Further, the liquid phase in the reactor is heated to a temperature of $65^{\circ} \mathrm{C}$, at which the reaction can already proceed. Upon reaching the temperature indicated above, acetylene start supplys in the reactor and bubble through the liquid phase. It should be noted that the reaction is carried out at atmospheric pressure, which greatly simplifies the design of the reactor unit.

The acetaldehyde formed during bubbling has a boiling point of $20.2^{\circ} \mathrm{C}$, due to which the reaction product evaporates from the reaction mass, the temperature of which is $65^{\circ} \mathrm{C}$. Thus, acetaldehyde, together with the unreacted part of the acetylene, is removed in gaseous form from the upper part of the reactor and enters the column for separating the reaction product. The column is partially filled with circulating ice water, which, absorbing acetaldehyde, goes to rectification, as a result of which the reaction product, acetaldehyde, is released. At the same time, unreacted acetylene is bubbled through the water in the column and is recycled. The water remaining after rectification is cooled and fed to the recirculation column for the separation of the reaction product.

Separately, it should be noted that the homogeneous catalyst in the reactor is solubled in water, and at a temperature of $65^{\circ} \mathrm{C}$ is not carried away along with the reaction product, but 
remains in the reactor. In this case, there is no need to separate the catalyst and return it to the reaction mass.

The proposed process is designed as a pilot-laboratory installation [3]. The process has several advantages. The reaction is doing at a low temperature and atmospheric pressure. There is no transfer of the catalyst along with the reaction product. Both the catalyst and the co-catalyst are present in the reaction mass in small amounts. No organic solvents are used in the process, and unreacted reagents are recycled, which makes the process accordingly with a principles of Green Chemistry.

Among the disadvantages of the process, it should be noted the need to ensure not only the heating of the reaction mixture to $65^{\circ} \mathrm{C}$, but also the need to cool the absorbing water to $5^{\circ} \mathrm{C}$.

\section{Conclusion}

The reaction of catalytic hydration of acetylene used in the described process using a metal complex catalyst based on gold (I) complex was researched using the reaction of catalytic hydration of phenylacetylene, as a result of which acetophenone was resieved. The conversion of 99\% and higher was achieved. The conversion level was determined from the intensity of the peaks in the ${ }^{1} \mathrm{H}$ NMR Spectra.

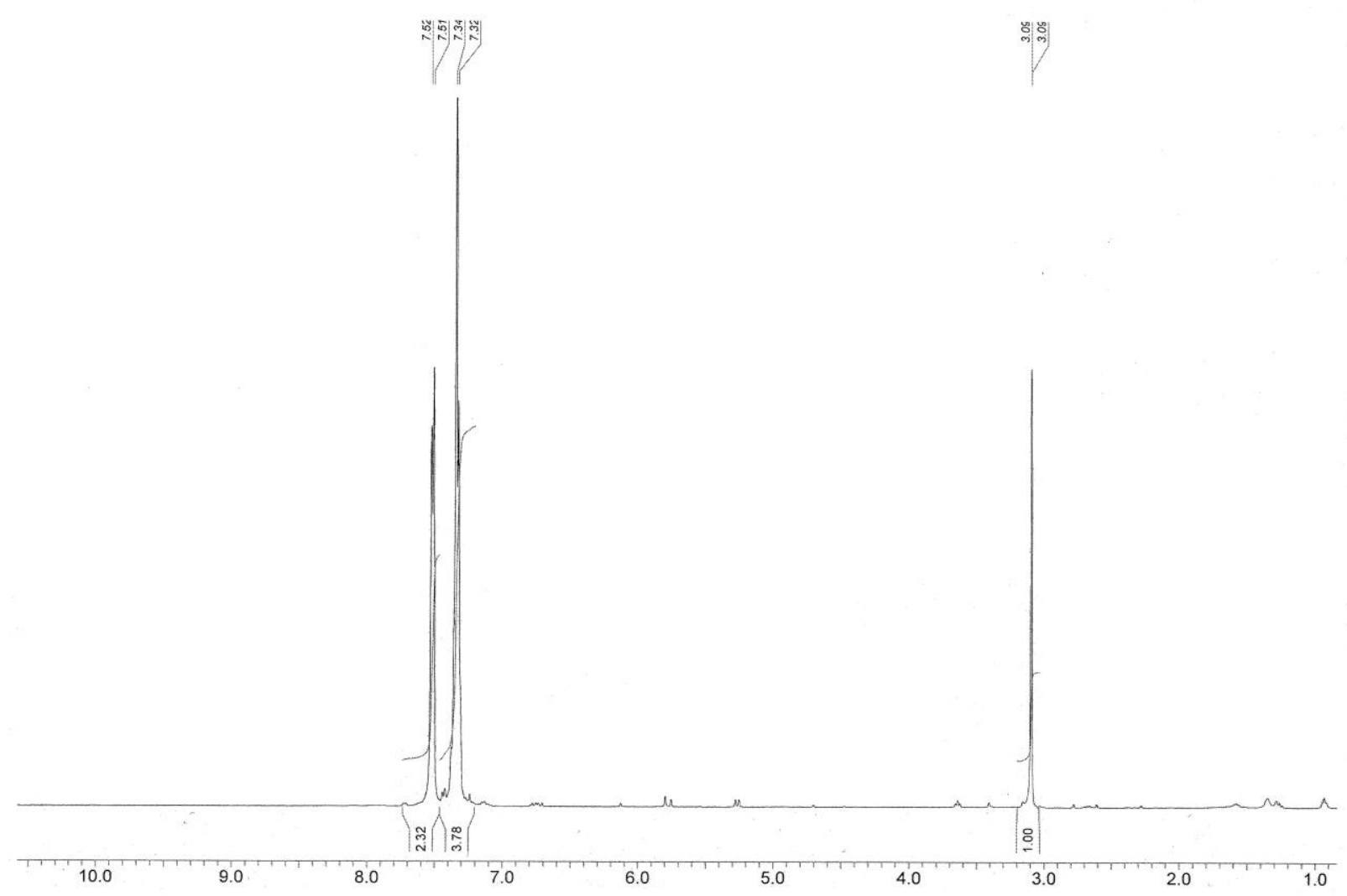

Fig. 2. ${ }^{1} \mathrm{H}$ NMR Spectra of the reaction mixture at the start of the reaction. 


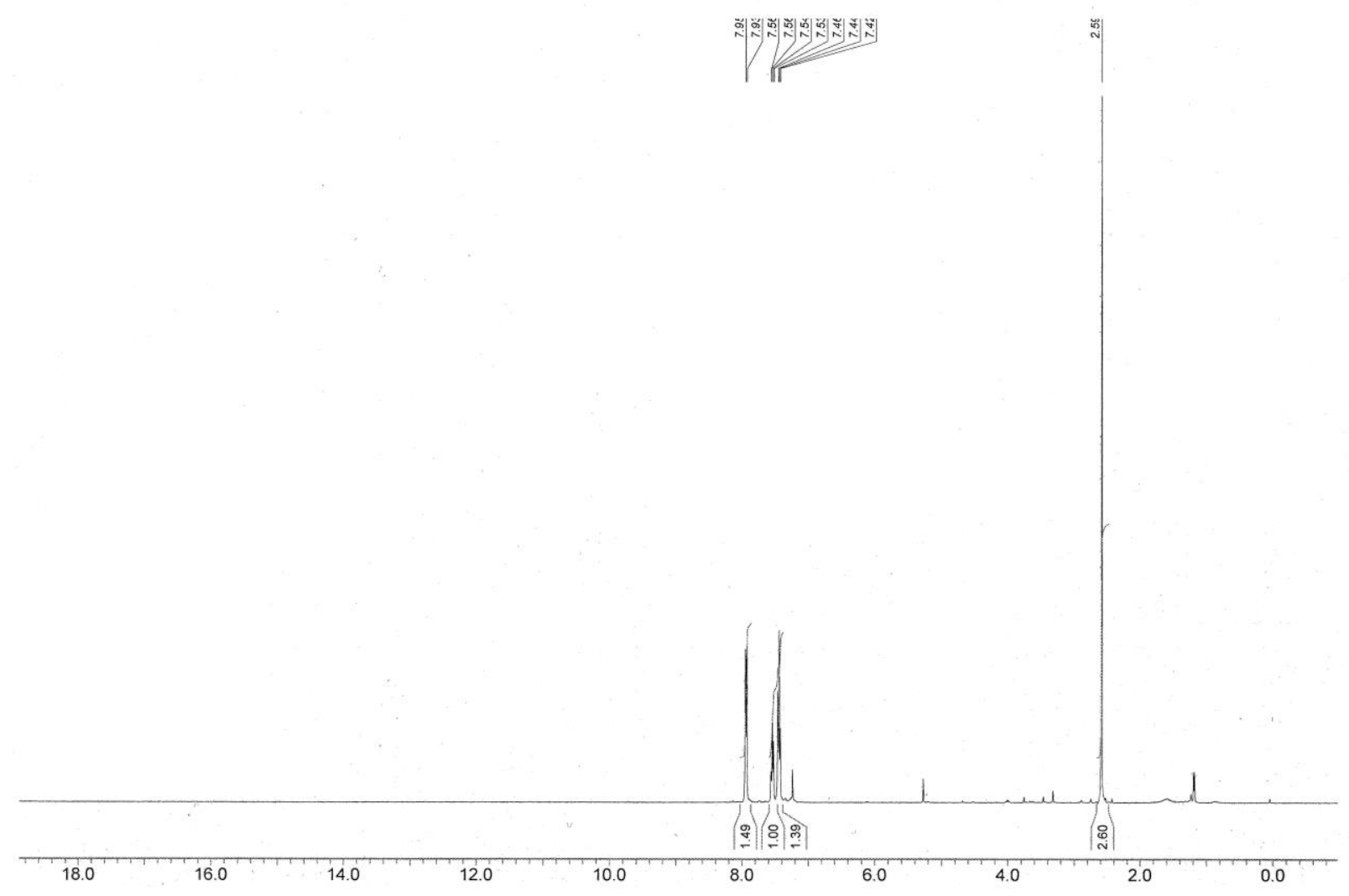

Fig.3. ${ }^{1} \mathrm{H}$ NMR Spectra of the reaction mixture at the end of the reaction.

To additional catalytic research the pilot-laboratory installation [3] is used, it is contained a gas-liquid reactor [2] and product separation column. Using this installation authors doing kinetic research the process described above. The presented process has a great perspectives for further research and industrial implementation.

\section{References}

[1] Sergio Sanz, Lathe A. Jones, Fabian Mohr, Mariano Laguna (2007). Homogenous Catalysis with Gold: Efficient Hydration of Phenylacetylene in Aqueous Media. Organometallics, 26, 952-957, https://doi.org/10.1021/om060821y

[2] Kokhan I.V., Gas-liquid reactor for processes using homogeneous metal complex catalysts. Technology-2019, The materials of the XXII International Scientific and Technical Conference, Sievierodonetsk.

[3] Kokhan I.V., The pilot-laboratory installation for the research of catalytic hydration of acetylene using a homogeneous metal complex catalyst. Technology-2020, The materials of the XXIII International Scientific and Technical Conference, Sievierodonetsk.

[4] Don W. Green, Marylee Z. Southard (Copyright 2019). Perry's Chemical Engineers' Handbook (9th Edition). Mc Graw Hill Education.

[5] Bruce E. Poling, John M. Prausnitz, John P. O’Connell (Copyright 2001). The Properties Of Gases And Liquids (5th Edition). Mc Graw Hill. 